\title{
Expression of periostin in breast cancer cells
}

\author{
KATARZYNA RATAJCZAK-WIELGOMAS ${ }^{1}$, JEDRZEJ GRZEGRZOLKA ${ }^{1}$, ALEKSANDRA PIOTROWSKA ${ }^{1}$, \\ RAFAL MATKOWSKI $^{3,4}$, ANDRZEJ WOJNAR ${ }^{3}$, JANUSZ RYS ${ }^{5}$, MACIEJ UGORSKI ${ }^{6}$ and PIOTR DZIEGIEL ${ }^{1,2}$ \\ ${ }^{1}$ Department of Histology and Embryology, Wroclaw Medical University, 50-368 Wroclaw; ${ }^{2}$ Department of Physiotherapy, \\ Wroclaw University School of Physical Education, 51-612 Wroclaw; ${ }^{3}$ Breast Unit, Department of Surgical Oncology, \\ Lower Silesian Oncology Centre; ${ }^{4}$ Department of Oncology and Surgical Oncology, Wroclaw Medical University, \\ 53-413 Wroclaw; ${ }^{5}$ Department of Tumour Pathology, Maria Sklodowska-Curie Memorial Cancer Center and \\ Institute of Oncology, 31-115 Krakow; ${ }^{6}$ Department of Biochemistry, Pharmacology and Toxicology, \\ Faculty of Veterinary Medicine, University of Environmental and Life Sciences, 50-375 Wroclaw, Poland
}

Received April 25, 2017; Accepted July 28, 2017

DOI: $10.3892 /$ ijo.2017.4109

\begin{abstract}
Periostin (POSTN) is a protein involved in multiple processes important for cancer development, both at the stage of cancer initiation and progression, as well as metastasis. The aim of this study was to determine the expression of POSTN in the cells of non-invasive ductal breast carcinoma in situ (DCIS) and invasive ductal carcinoma (IDC) and to correlate it with clinicopathological data. Immunohistochemical studies (IHC) were conducted on 21 cases of fibrocystic breast change (FC), 44 cases of DCIS and 92 cases of IDC. POSTN expression at mRNA (real-time PCR) and protein level (western blot analysis) was also confirmed in selected breast cancer cell lines (MCF-7, SK-BR-3, MDA-MB-231 and BO2). Statistically significant higher level of POSTN expression in IDC and DCIS cancer cells compared to FC was noted. Also, the level of POSTN expression in the cytoplasm of IDC cells was shown to increase with the increasing degree of tumour malignancy $(G)$ and significantly higher expression of POSTN was observed in each degree of tumour malignancy $(G)$ relative to FC. Statistically significant higher POSTN expression was observed in tumours with estrogen receptor-negative (ER') and progesterone receptor-negative ( $\left.\mathrm{PR}^{-}\right)$phenotypes in comparison to estrogen receptor-positive $\left(\mathrm{ER}^{+}\right)$and progesterone receptor-positive $\left(\mathrm{PR}^{+}\right)$cases, as well as significant negative correlation between POSTN expression in cancer cells and expression of ER and PR $(\mathrm{p}<0.05)$. Additionally, statistically significant differences in POSTN expression were shown between particular breast cancer cell lines, both at mRNA and protein level. Observed POSTN expression was the lowest in
\end{abstract}

Correspondence to: Dr Katarzyna Ratajczak-Wielgomas, Department of Histology and Embryology, Wroclaw Medical University, Chalubinskiego 6a, 50-368 Wroclaw, Poland

E-mail: katarzyna.ratajczak-wielgomas@umed.wroc.pl

Key words: periostin, cancer cell, breast cancer the case of MCF-7, and the highest in MDA-MB-231 and BO2 of the most aggressive potential clinically corresponding to $\mathrm{G} 3$ tumours. POSTN expression in the cytoplasm of IDC cancer cells may play an important role in cancer transformation mechanism.

\section{Introduction}

Periostin (POSTN) is a glycoprotein (93.3 kDA) that belongs to the group of so-called matricellular proteins (1) and it is involved in both physiological and pathological processes. Under physiological conditions, it plays an important role in collagen fibrillogenesis (2), cell adhesion and wound healing process (1). POSTN is also functionally important in the physiological process of epithelial-mesenchymal transition (EMT) that is crucial to normal embryonic development (3). It participates in the formation and maintenance of correct bone and teeth structure (4), and in heart development (atrioventricular valve) (5). In recent years, there have been a number of studies showing unique role of POSTN in oncogenesis process. The mechanism by which POSTN interacts with tumours has not been completely elucidated. Initial studies show that POSTN influences cancer cells by binding integrin receptors $\alpha v \beta_{3}$, $\alpha v \beta_{5}$ and $\alpha 6 \beta_{4}$ that are present on the surface of cancer cells and play an important role in cancer progression by stimulating angiogenesis and metastasis (6-11). This is supported by studies by Zhang et al (12) and Bao et al (13), who demonstrated that in breast and colorectal cancer POSTN interacts with integrins $\alpha v \beta_{3}$ and $\alpha v \beta_{5}$, thus inducing AKT/PKB signalling pathway. Furthermore, Zhu et al (14) demonstrated that recombinant POSTN promotes adhesion and migration of epithelial ovarian tumour cells, and that this function was inhibited by the $\alpha v \beta_{3}$ or $\alpha v \beta_{5}$ antibody, indicating that POSTN is important in the $\alpha v \beta_{3}$ or $\alpha v \beta_{5}$ integrin-dependent adhesion and migration of epithelial cells. Baril et al (15) showed, in turn, that in pancreatic cancer cells, the $\alpha 6 \beta_{4}$-integrin complex acts as the cell receptor of POSTN and this interaction promotes migration through phosphorylation of focal adhesion kinase (FAK) and protein kinase B (AKT) through activation of the PI3 kinase pathway. Moreover, Malanchi et al (16) found that POSTN 
recruits Wnt ligands and therefore increases Wnt signalling pathway in cancer stem cell population. Noteworthy are also studies by Lee et al (17), who were first to describe that inhibition of POSTN with a DNA aptamer may significantly inhibit potential for invasive growth and metastasis in breast cancer due to blocking the binding of POSTN to integrins and inhibiting the POSTN-induced signaling pathways.

Because of high POSTN expression in cancer cells, currently a great deal of attention is paid to possibilities of use of this protein as a diagnostic marker or prognostic factor. High POSTN expression in patients with various type of cancer is usually associated with poor prognosis. In those patients, significantly shorter survival time, increased risk of local lymph node involvement, metastasis, faster disease relapse and increased resistance to chemotherapy is observed (18-23). Similarly, in many in vitro studies on cancer cell lines, correlation between the level of POSTN expression and invasive phenotype and ability to metastasise was also shown $(13,15,24-26)$.

In our recently published study (27), as well as in studies of other authors (20), a different localization of POSTN expression in invasive ductal carcinoma (IDC) e.g. in cancer-associated fibroblasts (CAFs) and in cancer cells was confirmed. Using laser capture microdissection technique (LCM), we were first to show POSTN expression at mRNA level in both of those cell types as well as statistically significant higher expression of mRNA POSTN in stromal cells relative to cancer cells (27). Furthermore, significantly increased expression of POSTN in CAFs in IDC relative to pre-invasive ductal breast carcinoma in situ (DCIS) was also noted, which may indicate the role of POSTN expression by CAFs in breast cancer progression (27). In view of the above facts and as one of the very first, in this study we have attempted to examine the importance of POSTN expression in IDC cancer cells only, in reference to clinicopathological data.

\section{Materials and methods}

Patients and tissue samples. Immunohistochemical (IHC) studies were performed on 21 cases of fibrocystic breast change (FC) (control group), 44 paraffin-embedded samples of non-invasive DCIS and 92 archival paraffin blocks of invasive ductal carcinoma (IDC), diagnosed from 2000 to 2006. Paraffin blocks containing IDC were obtained from Lower Silesian Oncology Centre in Wroclaw, and samples of DCIS and FC were obtained from the Department of Tumour Pathology of the Maria Sklodowska-Curie Institute of Oncology in Krakow. The paraffin sections, stained with haematoxylin and eosin (H\&E), were used to verify the diagnosis and the malignancy grade of the tumours $(\mathrm{G})$, according to WHO criteria (28). The clinical and pathological traits of the patients are presented in Table I. The molecular subtypes were luminal A (45\%), luminal B (17\%), HER2 type (24\%). Triple-negative carcinoma was $14 \%$. The patients were treated by mastectomy or quadrantectomy, with a subsequent axillary lymph node resection. In 6 patients $(6.5 \%)$ neoadjuvant chemotherapy prior to surgical resection of the tumours was applied. Forty-eight patients $(51.6 \%)$ received adjuvant chemotherapy, whereas radiotherapy was administered to 39 (41.9\%). The mean age of the patients at diagnosis was $59.4 \pm 11.31$ years
Table I. Patients and tumour characteristics of invasive ductal cancer (IDC) cases.

\begin{tabular}{|c|c|c|}
\hline Parameters & No. & $(\%)$ \\
\hline \multicolumn{3}{|l|}{ Age (years) } \\
\hline$\leq 56$ & 42 & 45.6 \\
\hline$>56$ & 50 & 54.4 \\
\hline \multicolumn{3}{|l|}{ Tumour size } \\
\hline pT1 & 48 & 52.2 \\
\hline pT2 & 36 & 39.1 \\
\hline pT3 & 5 & 5.43 \\
\hline pT4 & 5 & 3.27 \\
\hline \multicolumn{3}{|l|}{ Grade } \\
\hline G1 & 11 & 12.0 \\
\hline $\mathrm{G} 2$ & 50 & 54.3 \\
\hline G3 & 31 & 33.7 \\
\hline \multicolumn{3}{|l|}{ Menopausal status } \\
\hline Pre & 24 & 26.0 \\
\hline Post & 68 & 74.0 \\
\hline \multicolumn{3}{|l|}{ Lymph nodes } \\
\hline No & 51 & 55.4 \\
\hline $\mathrm{N} 1, \mathrm{~N} 2, \mathrm{~N} 3$ & 39 & 42.4 \\
\hline NA & 2 & 2.2 \\
\hline \multicolumn{3}{|l|}{ ER } \\
\hline Positive & 70 & 76.0 \\
\hline Negative & 22 & 24.0 \\
\hline \multicolumn{3}{|l|}{ PR } \\
\hline Positive & 61 & 66.3 \\
\hline Negative & 31 & 33.7 \\
\hline \multicolumn{3}{|l|}{ HER-2 } \\
\hline Positive & 42 & 45.6 \\
\hline Negative & 50 & 54.4 \\
\hline \multicolumn{3}{|l|}{ POSTN } \\
\hline Positive (1-12 IRS) & 46 & 50 \\
\hline Negative (0 IRS) & 46 & 50 \\
\hline Luminal A & 41 & 45 \\
\hline Luminal B & 16 & 17 \\
\hline HER2 type & 22 & 24 \\
\hline Triple-negative (TN) & 13 & 14 \\
\hline
\end{tabular}

pT, pathologic size of tumour; G, grade of malignancy; pN, pathologic degree of regional lymph node spread; ER, estrogen receptor expression by IHC; PR, progesterone receptor expression by IHC; HER2, human epithelial growth factor receptor 2.

(range 33-84). The study was approved by the Commission of Bioethics of the Wroclaw Medical University, Poland. All the patients gave written informed consent to participate in the study.

Cell lines. Four human breast cancer cell lines, MCF-7, SK-BR-3 (Cell Lines Collection of the Ludwik Hirszfeld 
Institute of Immunology and Experimental Therapy, Wroclaw, Poland), MDA-MB-231 (ATCC, Washington, CO, USA) and BO2 (a derivative of MDA-MB-231, provided by Dr Philippe Clezardin; INSERM U664, Lyon, France) were used in this study. The MCF-7 cells represent the luminal epithelial phenotype exhibiting expression of estrogen receptor (ER), progesterone receptor (PR) and HER2 $\left(\mathrm{ER}^{+}, \mathrm{PR}^{+}\right.$and $\left.\mathrm{HER}^{+}\right)$ and are weakly invasive. The SK-BR-3 cells (ER-, $\mathrm{PR}^{+}$and HER $2^{+}$) are defined as weakly luminal epithelial-like, and are as invasive as the MCF-7 cells. The cells of MDA-MB-231 line and its derivative $\mathrm{BO} 2$ show no ER, PR or HER2 expression (ER- PR-, HER2-; triple-negative) but, instead, they express mesenchymal markers, including $\alpha$-smooth muscle actin ( $\alpha$-SMA). The latter cell lines show the highest metastatic potential as compared to the other two groups. All the breast cancer cells were cultured in $\alpha$-MEM medium supplemented with $10 \%$ fetal calf serum (FCS; Invitrogen, Carlsbad, CA, USA), $2 \mathrm{mM} \mathrm{L-glutamine} \mathrm{and} \mathrm{antibiotics.}$

$I H C$. Tumour samples were fixed in $4 \%$ buffered formalin, dehydrated and embedded in paraffin. Immunohistochemical reactions were performed using Autostainer Link 48 (Dako, Glostrup, Denmark). Deparaffinization and antigen retrieval were performed using Target Retrieval Solution, $\mathrm{pH} 9.0$ at $97^{\circ} \mathrm{C}$ for $20 \mathrm{~min}$ in a PT Link Rinse Station (both from Dako). The sections were washed in TBS/0.05\% Tween-20 and incubated with EnVision FLEX Peroxidase-Blocking reagent (5 min at RT). Then, the primary antibodies were applied for $20 \mathrm{~min}$ at RT. To detect POSTN expression, a rabbit polyclonal antibody was used (dilution 1:200; Novus Biologicals, Littleton, $\mathrm{CO}$, USA). The Ki-67 antigen was detected using mouse monoclonal antibody, clone MIB-1 (ready-to-use); estrogen receptors (ER) using mouse monoclonal antibody, clone 1D5 (diluted 1:100) and progesterone receptors (PR) using mouse monoclonal antibody, clone 636 (diluted 1:100) (both from Dako). The sections were then washed in TBS/0.05\% Tween-20 and EnVision FLEX/HRP secondary antibodies (Dako) were applied for $20 \mathrm{~min}$ at RT. The peroxidase substrate, diaminobenzidine (DAB), was applied and the sections were incubated for $10 \mathrm{~min}$ at RT. Finally, the sections were counterstained with Mayer's haematoxylin, dehydrated in alcohol (70, 96 and $99.8 \%$ ) and xylene, and mounted in the SUB-X mounting medium (Dako). HER2 expression status was determined using HercepTest ${ }^{\mathrm{TM}}$ and HER2 FISH pharmDx ${ }^{\mathrm{TM}}$ kit (both from Dako), according to the procedure recommended by the manufacturer. All reactions were conducted with negative controls, in which the primary antibodies were omitted.

RNA isolation, cDNA synthesis and real-time PCR. The total RNA from the cell lines under study was extracted using an RNeasy mini kit (Qiagen, Hilden, Germany), according to the manufacturer's protocol. To eliminate genomic DNA contamination, on-column DNase digestion was performed using RNase-Free DNase Set (Qiagen). The quantity and purity of RNA samples were assessed by measuring the absorbance at 260 and $280 \mathrm{~nm}$ with NanoDrop $1000 \mathrm{spec}-$ trophotometer (Thermo Fisher Scientific, Wilmington, DE, USA). Reverse transcription reactions were performed with the use of High-Capacity cDNA Reverse Transcription kits (Applied Biosystems, Foster City, CA, USA). The expression of POSTN mRNA in the studied cell lines was determined by real-time PCR with 7500 Real-time PCR system and iTag Universal Probes Supermix (Bio-Rad, Hercules, CA, USA) according to the manufacturer's protocol. $\beta$-actin was used as reference gene. For the reactions, the following sets of primers and TaqMan probes were used Hs00170815_m1 for POSTN, Hs99999903_m1 for $\beta$-actin (Applied Biosystems). All reactions were performed in triplicate under the following conditions: initial denaturation at $94^{\circ} \mathrm{C}$ for $10 \mathrm{~min}$ and 40 cycles of denaturation at $94^{\circ} \mathrm{C}$ for $15 \mathrm{sec}$, followed by annealing and elongation at $60^{\circ} \mathrm{C}$ for $160 \mathrm{sec}$. The relative expression of POSTN mRNA (RQ) was calculated with the $\Delta \Delta \mathrm{Ct}$ method (29).

Evaluation of IHC reactions. IHC reactions were assessed under a BX41 light microscope (Olympus) by two independent pathologists blinded to the experimental and patient data.

The expression of POSTN in cytoplasm was evaluated using the semi-quantitative IRS scale, according to Remmele and Stegner (30), which was used in our previous study (27). POSTN expression was evaluated on whole section (one slide per case). The scale is based on the percentage of tumour cells showing a positive reaction (A) (0 points, no cells with positive reaction; 1 point, $1-10 \%$ cells with positive reaction; 2 points, $11-50 \%$; 3 points, $51-80 \%$; 4 points, over $80 \%$ cells) as well as on the intensity of the reaction color (B) (0 points, no reaction; 1 point, low intensity; 2 points, moderate intensity; 3 points, strong intensity reaction color). The final score represents the product of the two values and falls in the range 0-12 (AxB). The nuclear expression of Ki-67 antigen was evaluated using a semi-quantitative five-grade scale based on the proportion of cells with the reaction product: ( 0 points, no reaction; 1 point, $1-10 \%$; 2 points, $11-25 \% ; 3$ points, $26-50 \%$; 4 points, over $50 \%$ of cells have the reaction product). The status of ER and PR receptors was scored from 0 to 3 points, depending on the percentage of positive cells ( 0 points, no reactions; 1 point, $1-10 \% ; 2$ points, $11-50 \%$; 3 points, $51-100 \%$ stained cells). The expression of HER2 receptors was evaluated using a scale that takes into account both the intensity of the membrane reaction and the percentage of positive tumour cells (31).

Western blot (WB) analysis. Whole cell lysates were obtained using a RIPA buffer (50 mM Tris-Cl pH 8.0, $150 \mathrm{mM} \mathrm{NaCl}$, $0.1 \%$ SDS, $1 \%$ Igepal CA-630), with the addition of a protease inhibitor cocktail, $0.5 \%$ sodium deoxycholate and $0.5 \mathrm{mM}$ phenylmethanesulfonylfluoride (PMSF) (all from Sigma, Munich, Germany). Protein concentration was measured using BCA technique (Thermo-Pierce, Rockford, IL, USA) and NanoDrop 1000 spectrophotometer (Thermo Fisher Scientific). Cell extracts were mixed with sample buffer $(250 \mathrm{mM}$ TRIS pH 6.8, 40\% glycerol, 20\% (v/v) b-mercaptoethanol, $100 \mathrm{mM}$ DTT, $0.33 \mathrm{mg} / \mathrm{ml}$ bromophenol blue, $8 \%$ SDS) and then subjected to denaturation for $10 \mathrm{~min}$ at $95^{\circ} \mathrm{C}$. Equal amounts of protein (30 $\mu \mathrm{g} / \mathrm{lane})$ were separated by electrophoresis in a 10\% gel, in Mini Protean 3 apparatus (Bio-Rad, Marnes-la-Coquette, France). Subsequently, the proteins were electrophoretically transferred to PVDF membrane (Immobilon P; Millipore, Billerica, MA, USA) and sites of non-specific binding were blocked using $3 \%$ BSA in TBST buffer. Next, for the detection of POSTN, the membranes 

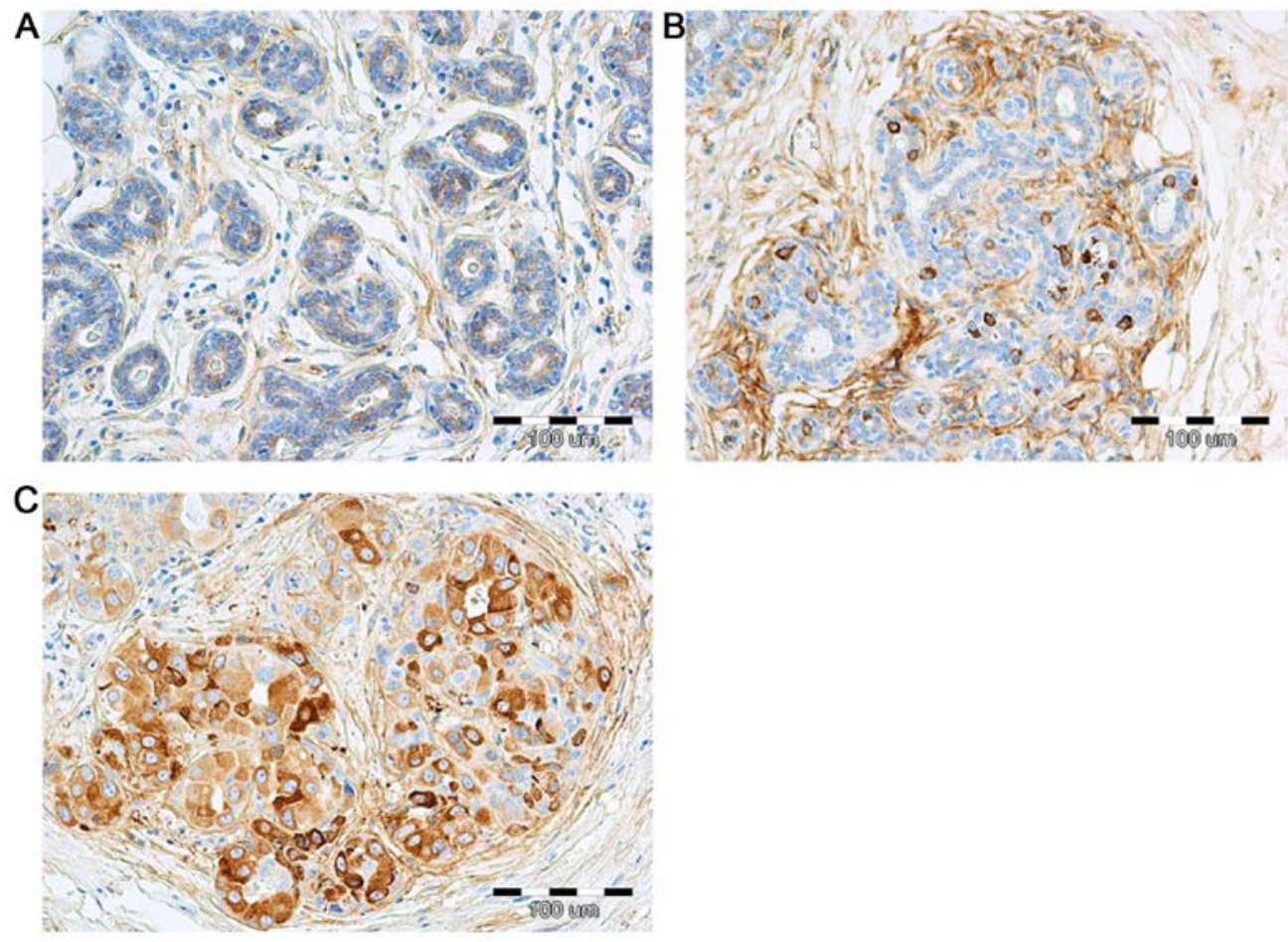

Figure 1. Expression of periostin (POSTN) in (A) fibrocystic change (FC) and in (B) cells of non-invasive ductal carcinoma in situ (DCIS) and in (C) invasive ductal breast carcinoma (IDC). Original magnification, x200.

were incubated with anti-human POSTN rabbit polyclonal antibodies (1:200; Novus Biologicals). Primary antibodies were incubated overnight at $4^{\circ} \mathrm{C}$. The membrane was rinsed in $0.2 \%$ TBST buffer and subsequently incubated for $1 \mathrm{~h}$ in a solution of donkey anti-rabbit antibody conjugated with peroxidase (1:2,000; Jacksons Immunoresearch, Suffolk, UK). The detection was conducted using a substrate for chemiluminescence (Immun-Star HRP Chemiluminescent kit; Bio-Rad) and the results were documented for exposure times ranging from $2 \mathrm{sec}$ to $30 \mathrm{~min}$ in Chemi-Doc XRS Molecular Imager apparatus (Bio-Rad). The resulting bands were estimated by densitometric quantitative analysis of proteins and normalized to $\beta$-tubulin (Abcam, Cambridge, UK) levels. The amount of the applied protein was controlled by staining the total protein on the membrane using Ponceau S (Sigma). POSTN expression was examined in three independent assays.

Immunofluorescence (IF). Cells were fixed with $4 \%$ paraformaldehyde for $12 \mathrm{~min}$ at RT and permeabilised using $0.2 \%$ Triton X-100 for $10 \mathrm{~min}$. The cells were incubated overnight at $4^{\circ} \mathrm{C}$ with primary anti-POSTN antibodies (1:200; Novus Biologicals). Subsequently, secondary fluorescein isothiocyanate (FITC)-conjugated anti-rabbit antibodies were applied (1:1,000, $1 \mathrm{~h}, \mathrm{RT})$ (Jacksons Immunoresearch). The sections were mounted in a DAPI-containing medium (ProLong Gold antifade reagent with DAPI) (Thermo Fisher Scientific) and analysed using a confocal laser scanning microscope FV3000 Fluoview (Olympus). Omitting the addition of primary antibody was performed as the respective negative controls.

Statistical analysis. Prism 5.0 software (GraphPad, La Jolla, CA, USA) was utilized to analyse the data. The Mann-Whitney test was used to compare the groups of data that did not meet the assumptions of the parametric test. Two-way ANOVA with the Bonferroni multiple comparison test was used to analyse the differences between the analysed cell lines. The correlations between the clinicopathological parameters and POSTN expression were analysed using the Mann-Whitney U test, Fisher's exact test and Spearman's correlation test. The Kaplan-Meier method and the log-rank test were used to analyse patients' overall survival. The cut-off scores of POSTN expression used for Kaplan-Meier overall survival curves were 0-2 vs. 3-12 pts. Cut-off points for the analysis were estimated based on median. For each variable, the hazard ratio (HR) and the $95 \%$ confidence interval $(95 \% \mathrm{CI})$ were estimated. In all analyses, the results were considered statistically significant when $\mathrm{p}<0.05$.

\section{Results}

Immunohistochemical POSTN expression and its impact on patients' clinicopathological data. In IHC experiments, POSTN expression in the cytoplasm of cancer cells was noticed in 46 cases of IDC (50\%). The IHC reaction showing POSTN expression in FC, DCIS and IDC cancer cells is presented in Fig. 1. The average value of POSTN expression in IDC cancer cells was $1.84 \pm 2.29$. Statistically significant higher level of POSTN expression in IDC cancer cells as compared to FC ( $\mathrm{p}<0.001$ Mann-Whitney U test) (Fig. 2) was observed. Also, significantly increased POSTN expression in DCIS vs. FC cells ( $p<0.005$, Mann-Whitney U test) (Fig. 2) was shown. Moreover, an increasing level of POSTN expression in the cytoplasm of IDC cells with the increase of the degree of tumour malignancy $(\mathrm{G})$ was shown in the analysed group of IDC cases, but no significant correlation was observed between particular malignancy grades. Also, significantly higher 

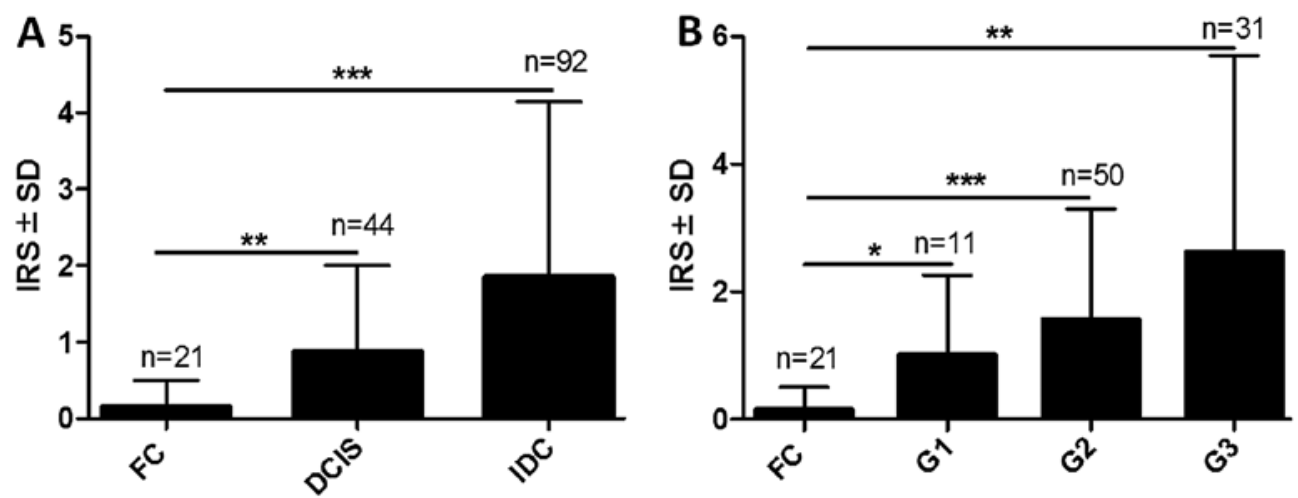

Figure 2. Differences between periostin (POSTN) expression in cancer cells in invasive ductal breast cancer (IDC), (n=92) and non-invasive ductal carcinoma in situ (DCIS), $(\mathrm{n}=44)$ compared to the control group $(\mathrm{FC}),(\mathrm{n}=21)$. (A) A statistically significant difference was noted between IDC vs. FC $\left({ }^{* * * *} \mathrm{p}<0.001\right)$, and DCIS vs. FC ( $*$ p $<0.005$, Mann-Whitney U test). Immunohistochemical POSTN expression in IDC tissues with regard to malignancy grade (G) compared to control group (FC). (B) Significantly higher POSTN expression was observed in G1 ( $\mathrm{n}=11), \mathrm{G} 2$ ( $\mathrm{n}=50)$ and G3 ( $\mathrm{n}=31)$ cases as compared to FC cases. ( $\mathrm{p}<0.05$, ${ }^{* *} \mathrm{p}<0.005$ and ${ }^{* * *} \mathrm{p}<0.001$, Mann-Whitney U test).

A
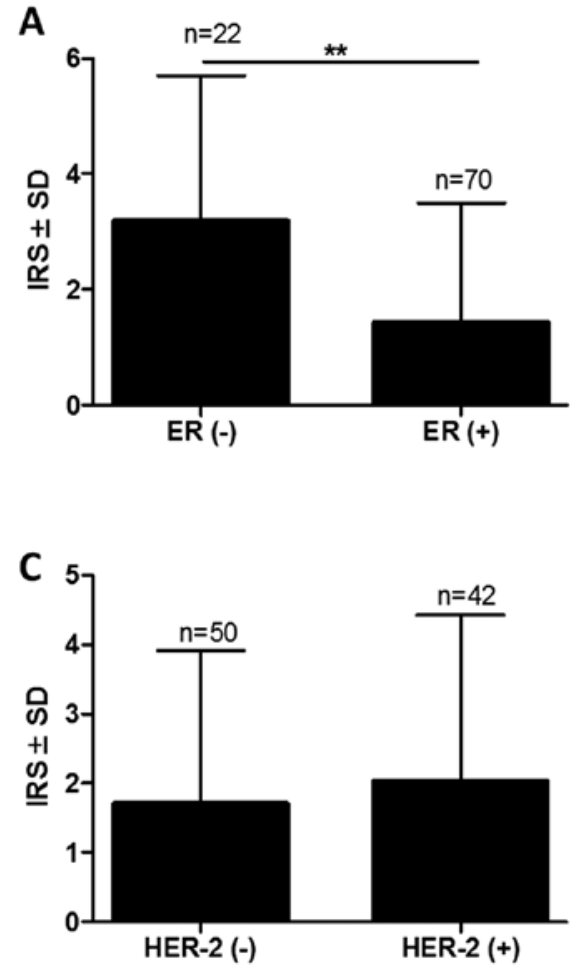

B
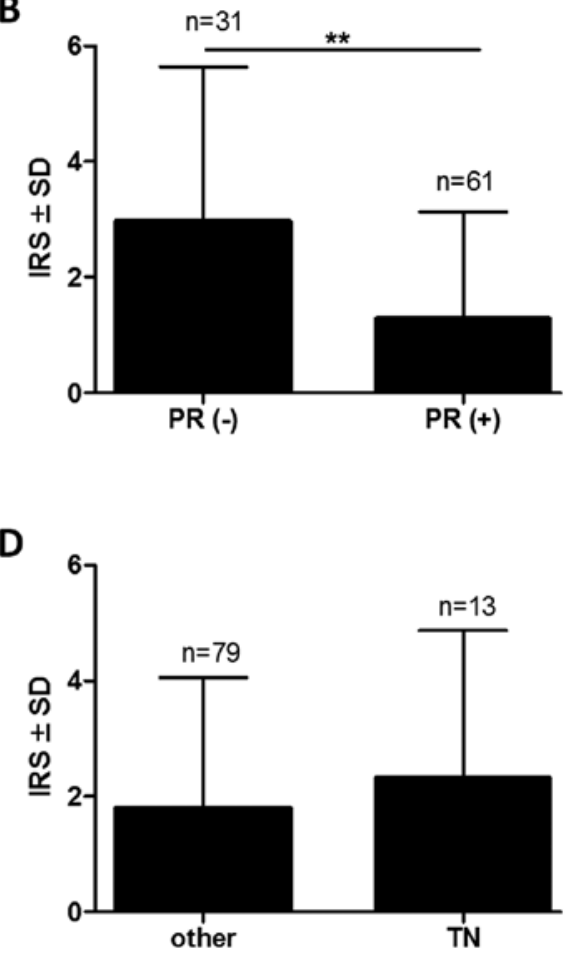

Figure 3. Correlation of periostin (POSTN) expression (IHC) in invasive ductal breast carcinoma (IDC) with clinicopathological factors. (A) Significantly higher POSTN expression levels were noted in estrogen receptor-negative $\left(\mathrm{ER}^{-}\right),(\mathrm{n}=22)$ as compared to estrogen receptor-positive $\left(\mathrm{ER}^{+}\right)$, $(\mathrm{n}=70) \mathrm{IDC}$ cases ( ${ }^{*} \mathrm{p}<0.005$, Mann-Whitney U test). (B) Significantly higher POSTN expression levels in PR negative $\left(\mathrm{PR}^{-}\right)$, $(\mathrm{n}=31)$ as compared to $P R$ positive $\left(\mathrm{PR}^{+}\right),(\mathrm{n}=61)$ tumours $\left({ }^{* *} \mathrm{p}<0.005\right.$, Mann-Whitney U test). (C) No significant association was noted in expression level of POSTN in HER2 negative (n=50) and HER2 positive cases $(n=42)$. (D) Triple-negative (TN; ER $\left./ \mathrm{PR}^{-} / \mathrm{HER}^{-}\right),(\mathrm{n}=13)$ were characterized by higher POSTN expression in comparison with other IDC subtypes, $(\mathrm{n}=79)$.

level of POSTN expression in particular degrees of tumour malignancy $(\mathrm{G})$ was found relative to its expression in cases of FC: FC vs. G1 (p=0.0111), FC vs. G2 (p<0.001), FC vs. G3 ( $\mathrm{p}<0.005)$, (Mann-Whitney U test) (Fig. 2). Statistically significant higher expression of POSTN in IDC cells in ER and $\mathrm{PR}^{-}$in comparison to $\mathrm{ER}^{+}$and $\mathrm{PR}^{+}(\mathrm{p}=0.0033, \mathrm{p}=0.0042$, Mann-Whitney U test) (Fig. 3) was also observed. No such significant correlation was found for HER2 antigen (Fig. 3). Also, higher POSTN expression was shown in the group of IDC tumours of triple negative phenotype $\left(\mathrm{ER}^{-}, \mathrm{PR}^{-}\right.$and HER2-), as relative to the group expressing those receptors, however, the correlation was not significant (Fig. 3). We also noted statistically significant negative correlation between cytoplasmic POSTN expression in IDC and the expression of ER and PR ( $r=-0.2488, p=0.0168 ; \mathrm{r}=-0.2062, \mathrm{p}=0.0486$, Spearman's rank correlation) (Fig. 4). Moreover, statistically significant positive correlation was observed between POSTN expression in IDC cancer cells and expression of Ki-67 proliferation antigen $(r=0.2042, p=0.0422$, Spearman's rank correlation) (Fig. 4).

In order to determine the importance of POSTN expression in the IDC tumour cells, analysis of correlation 

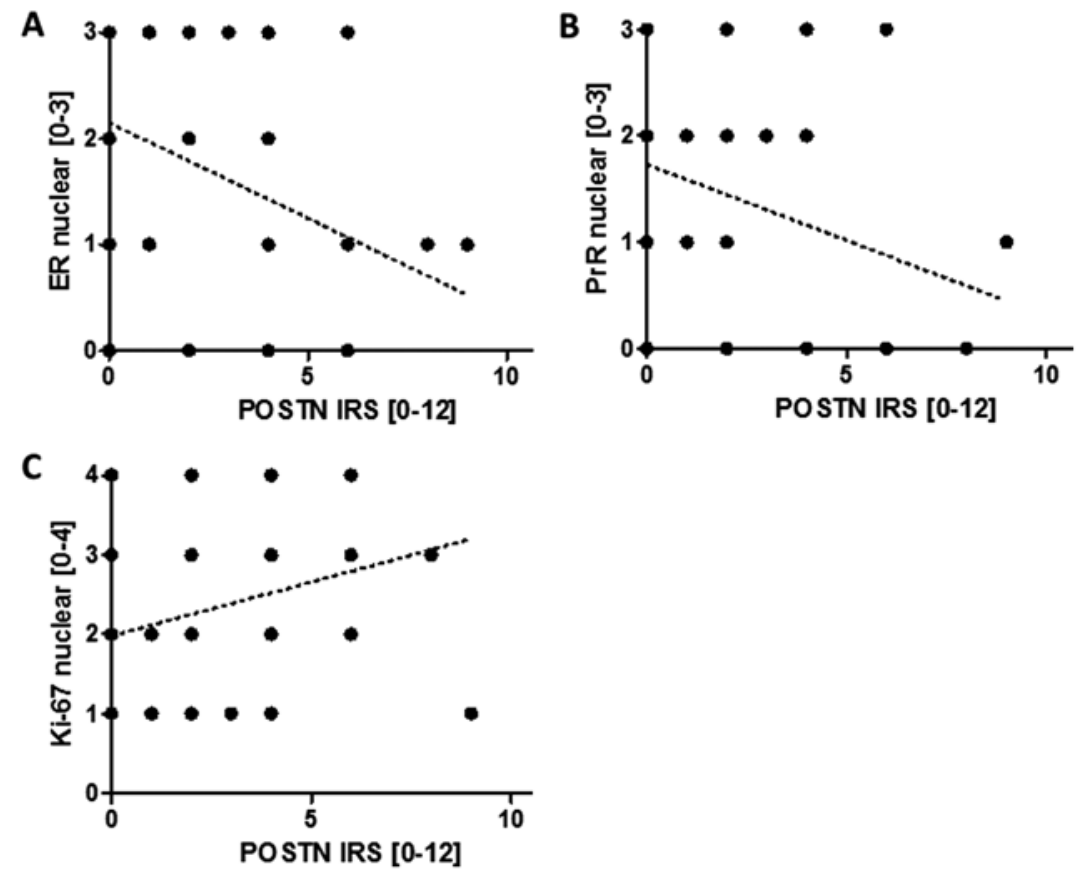

Figure 4. Significant negative correlation between cytoplasmic periostin (POSTN) expression and the expression of ER and PR in invasive ductal breast carcinoma (IDC) [(A) r=-0.2488, $\mathrm{p}=0.0168, \mathrm{~B}, \mathrm{r}=-0.2062, \mathrm{p}=0.0486$, Spearman's rank correlation]. Positive correlation between POSTN expression in cancer cell and nuclear expression of Ki-67 [(C) $\mathrm{r}=0.2042, \mathrm{p}=0.0422$, Spearman's rank correlation].

between obtained results and clinicopathological data was performed (Table II). With the use of $\chi^{2}$ test, in the analysed group of patients statistically significant correlation was shown between high level of POSTN expression in IDC cells ( $>2$ IRS points) and the expression of ER and $P R(p=0.015, p=0.0318$, Fisher's exact test) and lymph nodes metastases $(\mathrm{pN})(\mathrm{p}=0.049$, Fisher's exact test). However, no statistically significant correlation was observed between POSTN cytoplasmic expression in IDC and tumour malignancy grade (G), pre-menopausal stage, the size of primary tumour (pT) and age of patients.

Prognostic significance of POSTN expression. No statistically significant correlation between POSTN expression in cytoplasm of cancer cells and overall patients' survival time $(\mathrm{p}=0.287$, Mantel-Cox test) (Fig. 5). The results illustrate tendency towards shorter overall survival time (OS) in the group of patients with high POSTN expression (Fig. 5). Additionally, it was shown, that the presence of lymph node metastases $(\mathrm{pN})(\mathrm{p}<0.0001)$, tumour size $(\mathrm{p}<0.0001)$ and advanced disease stage $(\mathrm{p}<0.0001)$, (Mantel-Cox test) (Fig. 5) in the test group of patients were associated with patient poor OS.

Level of $M R N A$ and protein expression of POSTN in the examined cell lines. POSTN expression was also determined in vitro in the cells of selected breast cancer cell lines (MCF-7, SK-BR-3, MDA-MB-231 and BO2). With the use of real-time-PCR technique, statistically significant differences in mRNA expression of POSTN between particular cell lines were shown. The lowest expression of mRNA for POSTN was noted in the case of MCF-7 and SK-BR-3, and the highest in triple-negative cell lines (MDA-MB-231 and BO2): MCF-7 vs. MDA-MB-231 ( $\mathrm{p}<0.0001)$, MCF-7 vs. BO2 $(\mathrm{p}<0.0001)$, SK-BR-3 vs. MDA-MB-231 $(\mathrm{p}<0.001)$,
SK-BR-3 vs. BO2 ( $<$ 0.0001), (Bonferroni's multiple comparison test) (Fig. 6). Similar relation was observed also at the protein level. Differentiated level of expression of this glycoprotein was confirmed by the densitometric analysis of protein bands obtained on the membrane by WB. Analogically to the POSTN expression at the mRNA level, statistically higher level of protein POSTN expression was shown in MDA-MB-231 and $\mathrm{BO} 2$ cell lines in comparison with cells of MCF-7 and SK-BR-3 lines: MCF-7 vs. MDA-MB-231 ( $<<0.0001)$, MCF-7 vs. BO2 ( $<<0.0001)$, SK-BR-3 vs. MDA-MB-231 $(\mathrm{p}<0.0001)$, SK-BR-3 vs. BO2 $(\mathrm{p}<0.0001)$, (Bonferroni's multiple comparison test) (Fig. 6). Significant, strong correlation between POSTN expression in IDC cells at mRNA level and its expression on the protein level $(r=0.8833, p=0.0031$, Spearman's rank correlation) (Fig. 6) was also shown.

In order to verify POSTN localisation, immunofluorescence (IF) was performed on aforesaid breast cancer cell lines. Analysis of the results confirmed cytoplasmic nature of the reaction (Fig. 7) and is in line with the results of IHC studies.

\section{Discussion}

Existing literature data on the role of POSTN expression in breast cancer confirmed POSTN expression localized both in CAFs and in cancer cells, in which expression was usually found in 30-60\% of analysed cases of cancer $(19,20,27)$.

In the present study, for the first time, expression of POSTN localized only in cancer cells of pre-invasive DCIS and IDC were compared. POSTN expression in the cytoplasm of cancer cells was reported in 50\% of IDC cases by IHC technique. Statistically significant higher level of POSTN expression in IDC and DCIS compared to FC was recorded. Moreover, we observed an increasing level of POSTN expression in the cytoplasm of IDC cells with the increase of tumour malignancy 
Table II. Relationship between periostin (POSTN) expression in cancer cells and selected clinicopathological parameters in IDC patients.

\begin{tabular}{|c|c|c|c|c|}
\hline \multirow[b]{2}{*}{ Characteristics } & \multirow[b]{2}{*}{ No. $(\%)$} & \multicolumn{2}{|c|}{ POSTN expression, no. (\%) } & \multirow{2}{*}{$\begin{array}{c}\text { P-value } \\
\left(\chi^{2}\right)\end{array}$} \\
\hline & & (0-2 IRS) & (3-12 IRS) & \\
\hline \multicolumn{5}{|l|}{ Age (years) } \\
\hline$\leq 56$ & $42(45.6)$ & $25(27.2)$ & $19(20.6)$ & \\
\hline$>56$ & $50(54.4)$ & $25(27.2)$ & $23(25.0)$ & 0.68 \\
\hline \multicolumn{5}{|l|}{ Tumour size } \\
\hline pT1 & $48(52.2)$ & $26(28.2)$ & $22(24.0)$ & \\
\hline pT2 & $36(39.1)$ & $22(24.0)$ & $14(15.2)$ & \\
\hline pT3 & $5(5.43)$ & $1(1.1)$ & $4(4.3)$ & 0.311 \\
\hline pT4 & $5(3.27)$ & $1(1.1)$ & $2(2.1)$ & \\
\hline \multicolumn{5}{|l|}{ Grade } \\
\hline G1 & $11(12.0)$ & $8(8.7)$ & $3(3.2)$ & \\
\hline $\mathrm{G} 2$ & $50(54.3)$ & $25(27.2)$ & $25(27.2)$ & 0.39 \\
\hline G3 & $31(33.7)$ & $17(18.5)$ & $14(15.2)$ & \\
\hline \multicolumn{5}{|c|}{ Menopausal status } \\
\hline Pre & $24(26.0)$ & $17(18.5)$ & $7(7.6)$ & \\
\hline Post & $68(74.0)$ & $33(35.9)$ & $35(38.0)$ & 0.059 \\
\hline \multicolumn{5}{|l|}{ Lymph nodes } \\
\hline No & $51(55.4)$ & $22(24.0)$ & $29(31.5)$ & \\
\hline $\mathrm{N} 1, \mathrm{~N} 2, \mathrm{~N} 3$ & $39(42.4)$ & $27(29.3)$ & $12(13.0)$ & 0.049 \\
\hline $\mathrm{n} / \mathrm{A}$ & $2(2.2)$ & $1(1.1)$ & $1(1.1)$ & \\
\hline \multicolumn{5}{|l|}{ ER } \\
\hline Positive & $70(76.0)$ & 43 (46.7) & $27(29.4)$ & \\
\hline Negative & $22(24.0)$ & 7 (7.6) & $15(16.3)$ & 0.015 \\
\hline \multicolumn{5}{|l|}{ PR } \\
\hline Positive & $61(66.3)$ & $38(41.3)$ & $23(25.0)$ & \\
\hline Negative & $31(33.7)$ & $12(13.0)$ & $19(20.7)$ & 0.0318 \\
\hline \multicolumn{5}{|l|}{ HER-2 } \\
\hline Positive & $42(45.6)$ & $28(30.5)$ & $14(15.2)$ & 0.579 \\
\hline Negative & $50(54.4)$ & $36(39.1)$ & $14(15.2)$ & \\
\hline
\end{tabular}

Significant p-values are given in bold. IRS, immunoreactive score.

grade $(G)$. A similar trend was observed in our previous studies (27), which may indicate the significant role of POSTN localized in both stromal cells (CAFs) and cancer cells in the progression of breast cancer. Similarly to the results of our studies, Nuzzo et al (20), with the use of IHC technique, confirmed breast cancer expression of POSTN in both stromal cells and cytoplasm of cancer cells (40\% cases). The authors emphasised remarkably decreased expression of this protein in cancer cells in comparison to stromal cells, which is also in line with the tendency observed in our studies. Nuzzo et al (20) clearly suggested that reciprocal interactions between POSTN localized in cancer cells and in stromal cells may play a crucial role in breast carcinogenesis, most likely due to functional cross-talk between the two compartments (16,20,32-34). Noteworthy are also studies by Zhang et al (12), who by IHC technique obtained results that similarly to ours confirmed increased expression of POSTN in breast cancer in comparison to control group with benign breast lesions. However, in most of the cases studied, POSTN expression was localized mainly in the stroma of breast cancer, and only in few cases in the cytoplasm of cancer cells (12). This is in contrast to the results of our experiments, where cytoplasmic expression of POSTN was observed in $50 \%$ studied cancer cases. Those differences may be not only due to the different methodologies of research (usage of different antibodies), but also due to various quantities of studied breast cancer cases. Similarly, Puglisi et al (18) confirmed increased POSTN expression in breast cancer in comparison to corresponding normal tissues. In $57 \%$ of cases, POSTN expression was shown in the cytoplasm of cancer cells, which is in accordance with results obtained by us. These authors also reported significant correlation between cytoplasmic expression of POSTN and expression of progesterone receptors, which is also in line with the result of our IHC experiments. However, contrary to our studies, 

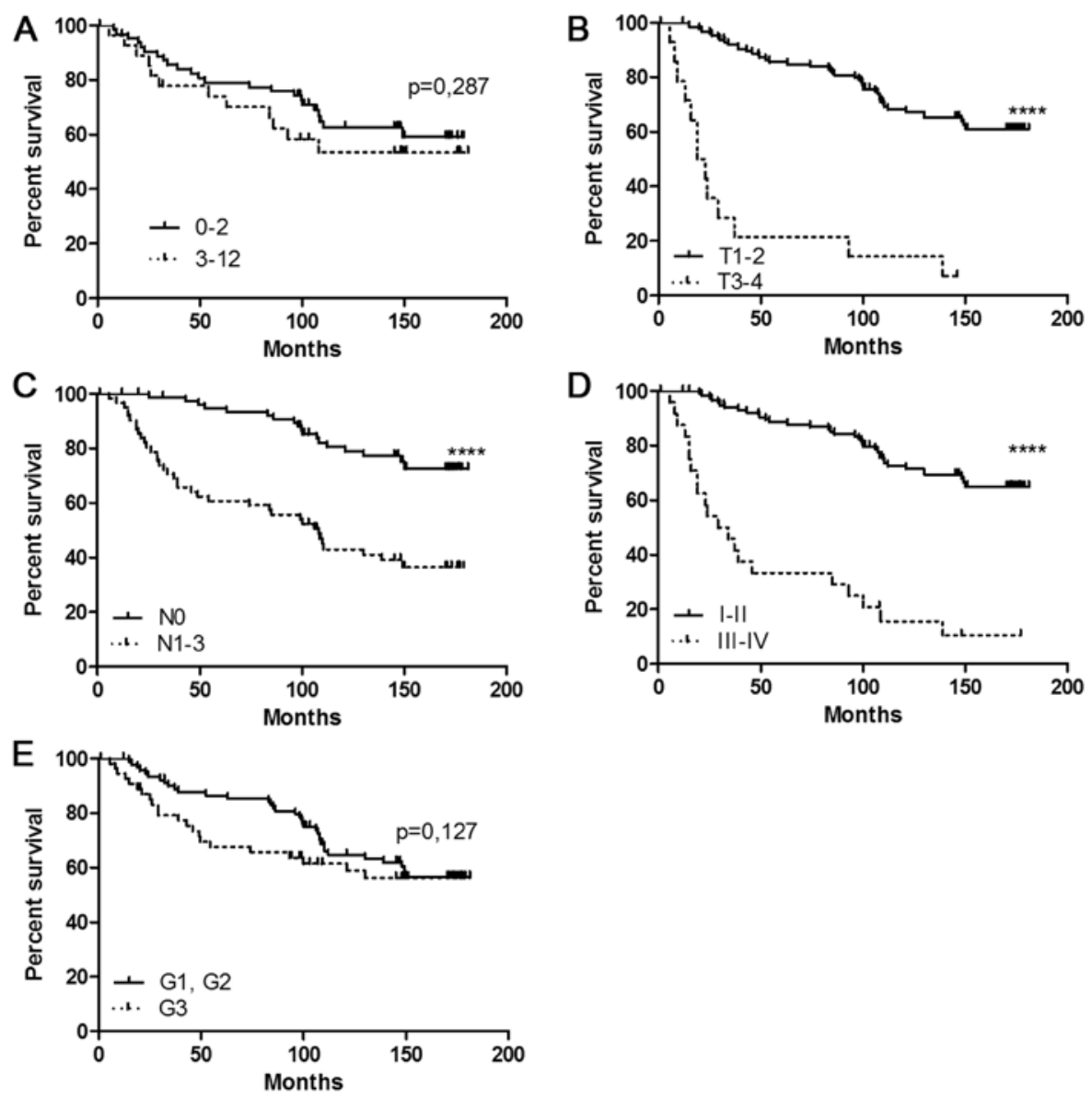

Figure 5. Kaplan-Meier survival curves in regard to (A) cytoplasmic periostin (POSTN) expression, (B) primary tumour size, (C) presence of lymph node metastases, (D) pTNM stage and (E) malignancy grade. ${ }^{* * * * *}$ p $<0.0001$.
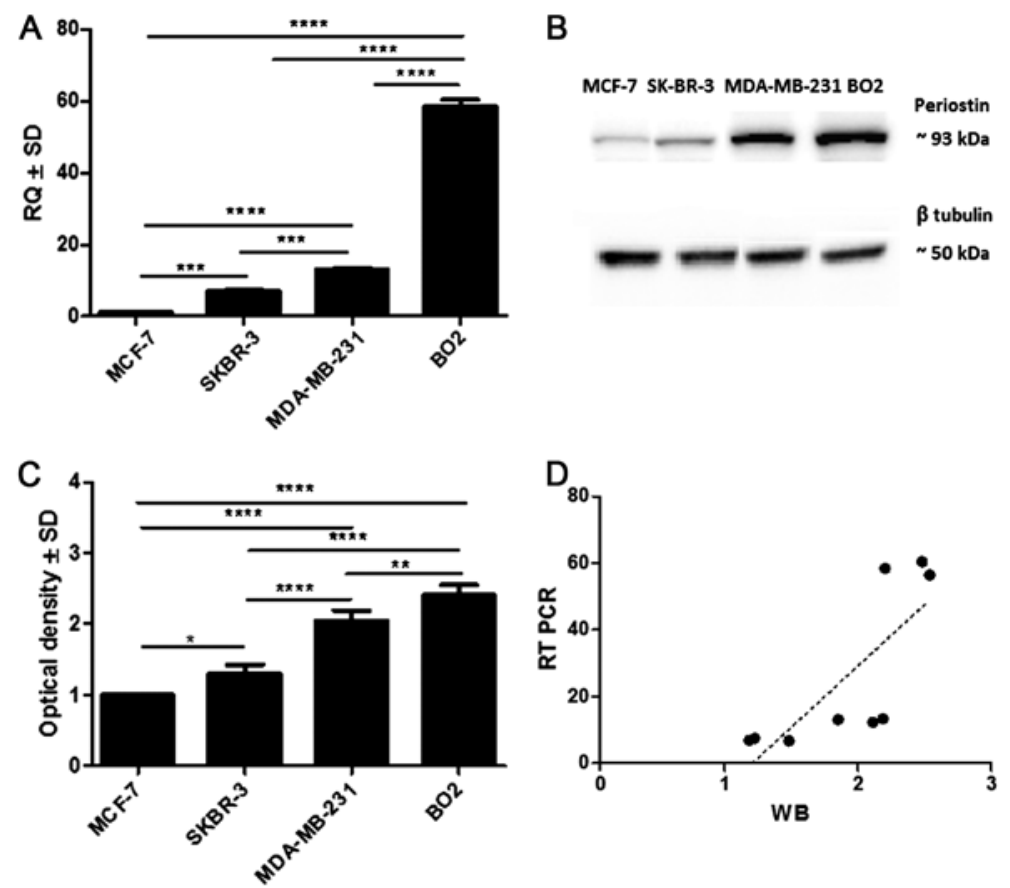

Figure 6. Characteristics of breast cancer cell lines according to periostin (POSTN) expression. (A) POSTN mRNA expression in MCF-7, SK-BR-3, MDA-MB-231 and BO2 breast cancer cell lines $\left({ }^{* * *} \mathrm{p}<0.001\right.$ and ${ }^{* * * *} \mathrm{p}<0.0001$, Bonferroni's multiple comparison test). Western blot bands in the membrane point to the presence of POSTN in studied cell lines. $\beta$-tubulin served as an internal control. (B) This blot is representative of three independent blots. Densitometric measurement of the bands demonstrates differences in POSTN protein contents between cell lines of MCF-7, SK-BR-3, MDA-MB-231 and BO2 . (C) A statistically significant difference was noted between the cell lines MCF-7, SK-BR-3 and MDA-MB-231 and BO2 ("p $<0.05$ and ${ }^{* * * *} \mathrm{p}<0.0001$, Bonferroni's multiple comparison test). OD, optical density; SD, standard deviation. (D) Positive correlation between cytoplasmic POSTN expression in IDC on the mRNA and protein level ( $\mathrm{r}=0.8833, \mathrm{p}=0.0031$, Spearman's rank correlation). 

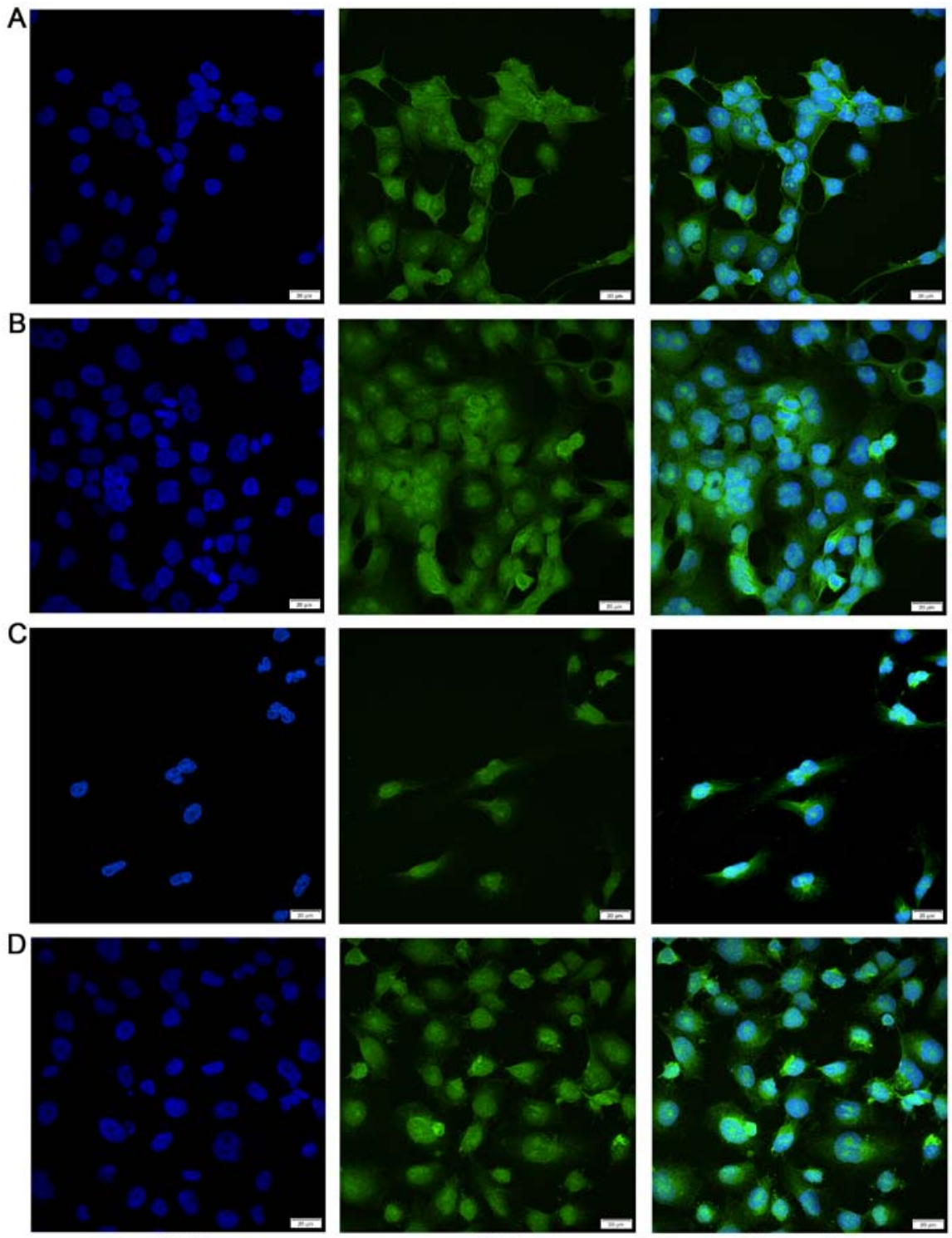

FITC

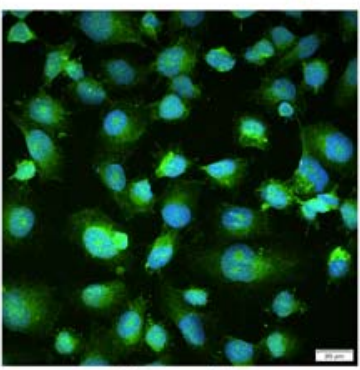

Merged

Figure 7. Cytoplasmic POSTN expression in breast cancer cell lines illustrated by immunofluorescence reaction, (A) MCF-7, (B) SK-BR-3, (C) MDA-MB-231 and (D) BO2.

no correlation between cytoplasmic expression of POSTN and the expression of Ki-67 proliferation antigen, and status of estrogen receptors, was shown. Moreover, in $12 \%$ cancer cases Puglisi et al also observed nuclear expression of studied glycoprotein (18), however, no such POSTN localization was confirmed in any other publication so far. Nuclear localization of POSTN shown in the study of Puglisi et al (18) seems quite surprising, as this glycoprotein is believed to be a component of extracellular matrix. Similar conclusions were drawn by Shao et al (35) by the analysis of POSTN expression in patients with breast cancer. With the use of WB and IHC, the authors confirmed increased expression of POSTN in the cytoplasm of cancer cells in comparison to the corresponding control tissues, indicating a role of POSTN in cancerous transformation, which is consequently in line with the results of our studies. Comparable results of IHC studies were obtained by the research group of $\mathrm{Xu}$ et al (19), who showed significantly higher POSTN expression in breast cancer in comparison to atypical hyperplasia. The authors observed POSTN expression in the cytoplasm of cancer cells ( $30 \%$ of cases), which is also in line with our results. Positive correlation was also found between POSTN expression and lymph node metastases (pN), as well as cancers with triple-negative (ER', $\mathrm{PR}^{-}$and HER2-) phenotype, which agrees with the tendency noticed by us. Those results, similarly to this study, show potential role of this glycoprotein in cancers that are more aggressive (19).

In this study, an attempt was made to evaluate intensity of POSTN expression in selected breast cancer cell lines (MCF-7, SK-BR-3, MDA-MB-231 and BO2) by WB and real-time PCR. Statistically significant differences in POSTN expression were shown between particular breast cancer cell lines, both at mRNA and protein level. The lowest observed POSTN expression was in MCF-7 (ER ${ }^{+}$, and the highest in triple-negative cell lines (MDA-MB-231 and $\mathrm{BO} 2$ ) of the most aggressive potential clinically corresponding to G3 tumours. Similar correlation was also observed in IHC studies, showing increasing level of POSTN expression with the increase of malignancy grade $(\mathrm{G})$. Expression of POSTN on mRNA and 
protein level in breast cancer cell lines (MCF-7, MDA-468) was also shown by Puglisi et al (18). Analogically to the results of our studies, with the use of real-time PCR technique, those authors showed over 50-fold lower expression of POSTN mRNA in MCF-7 cell line in comparison to MDA-468 (18). However, different results were obtained by Contié et al study group (36), who did not find expression of POSTN mRNA in selected breast cancer cell lines, MDA-MB-231, MDA-BO2, BT474, T47D and ZR75. It is worth emphasising, that it is the first study, and so far not confirmed in the research of other authors, that shows no POSTN expression in in vitro model in the aforementioned breast cancer cell lines (36). Noteworthy are also studies by Shao et al (35), who used cell lines overexpressing POSTN, i.e. 293T, B16F1 and MDA-MB-231. Their research on SCID mouse xenografts confirmed aggressive phenotype of the cells and showed enhanced tumour growth in comparison with control cells, which confirms important role of POSTN in cancer progression and agrees with the tendency observed by us. Interesting conclusion were also drawn by Vardaki et al (37), who analysed molecular profile of exosomes in metastatic and non-metastatic breast cancer cell lines MDA-MB-231 and MCF-7. The authors showed, that POSTN was enriched in the MDA-MB-231-derived exosomes compared to the MCF-7 ones. Analogically, in a pilot study of breast cancer patients it was shown, that POSTN was enriched in exosomes from patients with lymph node metastasis compared to exosomes from patients with localized disease (37). Such results allow us to assume, that POSTN may be specific biomarker of metastatic breast cancer, which is also in line with our results and the studies of other authors $(19,35)$.

It is also noteworthy that in the available literature, there are also some studies showing a decreased expression of POSTN mRNA in some types of cancers, i.e. gastric (38) and bladder (39) cancers, as well as in cancer cell lines, inter alia in colorectal cancer cell line SW837, bladder cancer cell line T24 and cervical cancer HeLa, CaSki, SiHa and C33A (40) cell lines. It is contrary to the results of our studies, which unambiguously showed an increased expression of POSTN on mRNA and protein level in IDC and analysed breast cancer cell lines. There are few possible theories to explain this relationship. However, it is believed, that in vitro model may not fully reflect the complexity of neoplastic changes occurring in vivo. The above correlation may be also explained by the fact, that POSTN may function differently due to alternative splicing events at the C-terminal region, resulting in 8 different spliced transcripts. Furthermore, decreased POSTN expression in some types of cancers may be also associated with different regulation of some signalling pathways. It was confirmed in the study of Kim et al (39), who showed, that in bladder cancer POSTN suppresses cell invasiveness through the downregulation of Akt phosphorylation and the upregulation of E-cadherin via twist inhibition. Additionally, Lv et al (38) showed, that POSTN may function as a tumour suppressor in gastric cancer through stabilizing p53 and E-cadherin proteins via the $\mathrm{Rb} / \mathrm{E} 2 \mathrm{~F} 1 / \mathrm{p} 14 \mathrm{ARF} / \mathrm{Mdm} 2$ signaling pathway (38).

The aim of the present study, as one of the very few, was also to analyse the overall survival time of IDC patients depending on the intensity of POSTN expression in the cytoplasm of cancer cells. No statistically significant correlation was found between cytoplasmic expression of POSTN and overall patients' survival time. Only the tendency towards low survival rate in patients with high POSTN expression in the cytoplasm of cancer cells was observed. Slightly different results were obtained by Xu et al (19), who showed that high POSTN expression in cancer cells was significantly associated with a higher mortality risk. Additionally in the Cox regression test, POSTN protein was detected as an independent prognostic factor. Those differences may be due to the fact, that for this study we used significantly smaller pool of breast cancer cases. Nuzzo et al (20) showed also correlation between POSTN expression and a higher mortality risk and this may be also a result of conducting the analysis on significantly larger group of patients. The authors showed also different patients' mortality rate depending on the localization of POSTN expression, i.e. in stromal cells or in the cytoplasm of cancer cells. They found, that positive POSTN expression in cancer cells and low expression in stromal cells is associated with low patients' mortality rate as opposite to the group of patients, in which high POSTN expression was shown in those types of cells, or in the group of patients with low or absent POSTN expression in both cell compartments. According to the authors, this fact confirms functional cross-talk between both cell compartments (20).

In conclusion, this study is the first to cover the subject of POSTN expression in IDC and DCIS cells only and to describe the correlation with clinicopathological data. We showed increased POSTN expression in IDC cells relative to DCIS cells, which may indicate the role of POSTN expression in the IDC cancer cells in the process of cancerous transformation. Moreover, an increasing level of POSTN expression in cancer cells with the increase of tumour malignancy grade $(\mathrm{G})$ was also shown, as well as the significant negative correlation between POSTN expression in the cytoplasm of cancer cells, and ER and PR receptors. No such significant association was observed with POSTN expression by stromal cells (CAFs) (27). Those results may suggest, that POSTN localized in the cytoplasm of IDC cells may play a role in the regulation of expression of estrogen and progesterone receptors in IDC. Additionally, in the in vitro studies, we showed an increase in POSTN expression at mRNA and protein level in breast cancer cell lines representing particular phenotype and tumour malignancy grade. Therefore, it should be emphasised, that POSTN localized in the cytoplasm of cancer cells as well as in stromal cells (CAFs) (27) may be a factor playing an important role in IDC, particularly in the process of cancer progression. Based on the presented results as well as on our previous studies (27) and the existing data, may suggest crosstalk between stromal cells (CAFs) and cancer cells in IDC, which is also in line with the results Nuzzo et al (20).

In conclusion, POSTN may be a promising subject of research aimed at searching for alternative solutions in breast cancer therapy.

\section{References}

1. Hamilton DW: Functional role of periostin in development and wound repair: Implications for connective tissue disease. J Cell Commun Signal 2: 9-17, 2008.

2. Norris RA, Damon B, Mironov V, Kasyanov V, Ramamurthi A, Moreno-Rodriguez R, Trusk T, Potts JD, Goodwin RL, Davis J, et al: Periostin regulates collagen fibrillogenesis and the biomechanical properties of connective tissues. J Cell Biochem 101: 695-711, 2007. 
3. Lindsley A, Snider P, Zhou H, Rogers R, Wang J, Olaopa M, Kruzynska-Frejtag A, Koushik SV, Lilly B, Burch JB, et al: Identification and characterization of a novel Schwann and outflow tract endocardial cushion lineage-restricted periostin enhancer. Dev Biol 307: 340-355, 2007.

4. Horiuchi K, Amizuka N, Takeshita S, Takamatsu H, Katsuura M, Ozawa H, Toyama Y, Bonewald LF and Kudo A: Identification and characterization of a novel protein, periostin, with restricted expression to periosteum and periodontal ligament and increased expression by transforming growth factor beta. J Bone Miner Res 14: 1239-1249, 1999.

5. Conway SJ and Molkentin JD: Periostin as a heterofunctional regulator of cardiac development and disease. Curr Genomics 9: 548-555, 2008

6. Ruan K, Bao S and Ouyang G: The multifaceted role of periostin in tumorigenesis. Cell Mol Life Sci 66: 2219-2230, 2009.

7. Morra L and Moch H: Periostin expression and epithelial-mesenchymal transition in cancer: A review and an update. Virchows Arch 459: 465-475, 2011

8. Ratajczak-Wielgomas K and Dziegiel P: The role of periostin in neoplastic processes. Folia Histochem Cytobiol 53: 120-132, 2015.

9. Kudo Y, Siriwardena BS, Hatano H, Ogawa I and Takata T: Periostin: Novel diagnostic and therapeutic target for cancer Histol Histopathol 22: 1167-1174, 2007.

10. Nuzzo PV, Buzzatti G, Ricci F, Rubagotti A, Argellati F, Zinoli L and Boccardo F: Periostin: A novel prognostic and therapeutic target for genitourinary cancer? Clin Genitourin Cancer 12 : 301-311, 2014

11. Siriwardena BS, Kudo Y, Ogawa I, Kitagawa M, Kitajima S, Hatano H, Tilakaratne WM, Miyauchi M and Takata T: Periostin is frequently overexpressed and enhances invasion and angiogenesis in oral cancer. Br J Cancer 95: 1396-1403, 2006.

12. Zhang Y, Zhang G, Li J, Tao Q and Tang W: The expression analysis of periostin in human breast cancer. J Surg Res 160: 102-106, 2010.

13. Bao S, Ouyang G, Bai X, Huang Z, Ma C, Liu M, Shao R, Anderson RM, Rich JN and Wang XF: Periostin potently promotes metastatic growth of colon cancer by augmenting cell survival via the Akt/PKB pathway. Cancer Cell 5: 329-339, 2004

14. Zhu M, Fejzo MS, Anderson L, Dering J, Ginther C, Ramos L, Gasson JC, Karlan BY and Slamon DJ: Periostin promotes ovarian cancer angiogenesis and metastasis. Gynecol Oncol 119: 337-344, 2010

15. Baril P, Gangeswaran R, Mahon PC, Caulee K, Kocher HM, Harada T, Zhu M, Kalthoff $\mathrm{H}$, Crnogorac-Jurcevic $\mathrm{T}$ and Lemoine NR: Periostin promotes invasiveness and resistance of pancreatic cancer cells to hypoxia-induced cell death: Role of the beta4 integrin and the PI3k pathway. Oncogene 26: 2082-2094, 2007.

16. Malanchi I, Santamaria-Martínez A, Susanto E, Peng H, Lehr HA, Delaloye JF and Huelsken J: Interactions between cancer stem cells and their niche govern metastatic colonization. Nature 481 85-89, 2011.

17. Lee YJ, Kim IS, Park SA, Kim Y, Lee JE, Noh DY, Kim KT, Ryu SH and Suh PG: Periostin-binding DNA aptamer inhibits breast cancer growth and metastasis. Mol Ther 21: 1004-1013, 2013.

18. Puglisi F, Puppin C, Pegolo E, Andreetta C, Pascoletti G, D'Aurizio F, Pandolfi M, Fasola G, Piga A, Damante G, et al: Expression of periostin in human breast cancer. J Clin Pathol 61: 494-498, 2008.

19. Xu D, Xu H, Ren Y, Liu C, Wang X, Zhang H and Lu P: Cancer stem cell-related gene periostin: A novel prognostic marker for breast cancer. PLoS One 7: e46670, 2012

20. Nuzzo PV, Rubagotti A, Zinoli L, Salvi S, Boccardo S and Boccardo F: The prognostic value of stromal and epithelial periostin expression in human breast cancer: Correlation with clinical pathological features and mortality outcome. BMC Cancer 16: 95, 2016

21. Tian B, Zhang Y and Zhang J: Periostin is a new potential prognostic biomarker for glioma. Tumour Biol 35: 5877-5883, 2014.
22. Tian Y, Choi CH, Li QK, Rahmatpanah FB, Chen X, Kim SR, Veltri R, Chia D, Zhang Z, Mercola D, et al: Correction: Overexpression of periostin in stroma positively associated with aggressive prostate cancer. PLoS One 10: e0130333, 2015.

23. Li JS, Sun GW, Wei XY and Tang WH: Expression of periostin and its clinicopathological relevance in gastric cancer. World J Gastroenterol 13: 5261-5266, 2007

24. Tai IT, Dai M and Chen LB: Periostin induction in tumor cell line explants and inhibition of in vitro cell growth by anti-periostin antibodies. Carcinogenesis 26: 908-915, 2005.

25. Gillan L, Matei D, Fishman DA, Gerbin CS, Karlan BY and Chang DD: Periostin secreted by epithelial ovarian carcinoma is a ligand for alpha(V)beta(3) and alpha(V)beta(5) integrins and promotes cell motility. Cancer Res 62: 5358-5364, 2002.

26. Liu Y and Liu BA: Enhanced proliferation, invasion, and epithelial-mesenchymal transition of nicotine-promoted gastric cancer by periostin. World J Gastroenterol 17: 2674-2680, 2011.

27. Ratajczak-Wielgomas K, Grzegrzolka J, Piotrowska A, Gomulkiewicz A, Witkiewicz W and Dziegiel P: Periostin expression in cancer-associated fibroblasts of invasive ductal breast carcinoma. Oncol Rep 36: 2745-2754, 2016.

28. Lakhani SR, Ellis IO, Schnitt SJ, Tan PH and van de Vijver MJ (eds): WHO Classification of Tumours of the Breast. 4th edition. IARC Press, Lyon, 2012.

29. Schmittgen TD and Livak KJ: Analyzing real-time PCR data by the comparative C(T) method. Nat Protoc 3: 1101-1108, 2008.

30. Remmele W and Stegner HE: Recommendation for uniform definition of an immunoreactive score (IRS) for immunohistochemical estrogen receptor detection (ER-ICA) in breast cancer tissue. Pathologe 8: 138-140, 1987 (In German).

31. Mueller-Holzner E, Fink $V$, Frede $T$ and Marth $C$ : Immunohistochemical determination of HER2 expression in breast cancer from core biopsy specimens: A reliable predictor of HER2 status of the whole tumor. Breast Cancer Res Treat 69 : $13-19,2001$

32. Nuzzo PV, Rubagotti A, Argellati F, Di Meglio A, Zanardi E, Zinoli L, Comite P, Mussap M and Boccardo F: Prognostic value of preoperative serum levels of periostin (PN) in early breast cancer (BCa). Int J Mol Sci 16: 17181-17192, 2015.

33. Erkan M,Kleeff J, Gorbachevski A, Reiser C,Mitkus T, Esposito I, Giese T, Büchler MW, Giese NA and Friess H: Periostin creates a tumor-supportive microenvironment in the pancreas by sustaining fibrogenic stellate cell activity. Gastroenterology 132: $1447-1464,2007$

34. Wang $Z$ and Ouyang G: Periostin: A bridge between cancer stem cells and their metastatic niche. Cell Stem Cell 10: 111-112, 2012.

35. Shao R, Bao S, Bai X, Blanchette C, Anderson RM, Dang T, Gishizky ML, Marks JR and Wang XF: Acquired expression of periostin by human breast cancers promotes tumor angiogenesis through up-regulation of vascular endothelial growth factor receptor 2 expression. Mol Cell Biol 24: 3992-4003, 2004.

36. Contié S, Voorzanger-Rousselot N, Litvin J, Clézardin P and Garnero P: Increased expression and serum levels of the stromal cell-secreted protein periostin in breast cancer bone metastases. Int J Cancer 128: 352-360, 2011.

37. Vardaki I, Ceder S, Rutishauser D, Baltatzis G, Foukakis T and Panaretakis T: Periostin is identified as a putative metastatic marker in breast cancer-derived exosomes. Oncotarget 7: 74966-74978, 2016.

38. Lv H, Liu R, Fu J, Yang Q, Shi J, Chen P, Ji M, Shi B and Hou P Epithelial cell-derived periostin functions as a tumor suppressor in gastric cancer through stabilizing p53 and E-cadherin proteins via the $\mathrm{Rb} / \mathrm{E} 2 \mathrm{~F} 1 / \mathrm{p} 14 \mathrm{ARF} / \mathrm{Mdm} 2$ signaling pathway. Cell Cycle 13: 2962-2974, 2014.

39. Kim CJ, Sakamoto K, Tambe Y and Inoue H: Opposite regulation of epithelial-to-mesenchymal transition and cell invasiveness by periostin between prostate and bladder cancer cells. Int J Oncol 38: 1759-1766, 2011.

40. Yoshioka N, Fuji S, Shimakage M, Kodama K, Hakura A, Yutsudo M, Inoue $\mathrm{H}$ and Nojima $\mathrm{H}$ : Suppression of anchorageindependent growth of human cancer cell lines by the TRIF52/periostin/OSF-2 gene. Exp Cell Res 279: 91-99, 2002. 